\title{
Upregulation of Brain-Derived Neurotrophic Factor (BDNF) in Patients With Mental Illness Not Using Psychotropic Medication
}

Hedda Soloey-Nilsen ( $\sim$ hedda.beate.soloy-nilsen@nordlandssykehuset.no )

Nordland hospital Trust

Kristin Nygaard-Odeh

Nordland hospital Trust

Magnhild Gangsoey Kristiansen

Nordland hospital Trust

Ole Lars Brekke

Nordland hospital Trust

Tom Eirik Mollnes

Nordland hospital Trust

Solveig Klaebo Reitan

Norwegian University of Science and Technology

\section{Terje Oiesvold}

Nordland hospital Trust

\section{Research Article}

Keywords: Brain derived neurotropic factor (BDNF), high-sensitive- C-reactive protein (hs-CRP), general psychiatric symptoms

Posted Date: November 1st, 2021

DOI: https://doi.org/10.21203/rs.3.rs-958122/v1

License: (c) (1) This work is licensed under a Creative Commons Attribution 4.0 International License. Read Full License 


\section{Abstract}

\section{Background}

There is evidence that brain-derived neurotropic factor (BDNF) plays a protective role in the brain. Peripheral levels of BDNF correlate with its concentration in the brain. Previous studies have revealed lower serum BDNF levels in patients with mental illnesses. In most studies serum BDNF correlates negatively with psychiatric disorders and disease severity. Most studies in this field are on psychiatric diagnosis and personality traits. The aim of our study is to explore associations between general psychiatric symptoms, independent of diagnostic groups, and serum BDNF as well as the inflammatory biomarker high-sensitive CRP (hs-CRP). Comparison between the group regularly using psychotropic medication and those not using psychotropic medication is conducted.

Methods

The study is a cross sectional study with 132 participants from a general open inpatient psychiatric ward at the Nordland Hospital Trust, Bodø, Norway. Participants were assessed on serum levels of BDNF and hs-CRP. Psychiatric symptoms were assessed by a self-rating scale (Symptom check list, SCL-90- R). Multiple linear regression model was used for statistical analyses of associations between levels of BDNF, hs-CRP and symptoms.

Results

We found a positive association $(p<0.05)$, for most SCL-90 symptom clusters with BDNF in the psychotropic medication-free group. No associations were found in the group of patients using psychotropic medication, except one, the Paranoid Ideation cluster $(p 0.022)$. No associations were found between hs-CRP and symptom clusters.

\section{Conclusion:}

We found no relation between symptom clusters and the inflammatory biomarker hs-CRP. Serum BDNF levels were positively associated with intensity of psychiatric symptoms in the group of patients not using psychotropic medication. In the group on psychotropic medication no associations for BDNF were seen. Our findings are in conflict with several previous studies reporting increased hs-CRP as well as decreased rather than increased BDNF in mental suffering.

\section{Introduction}

Identifying the causative and maintaining role of biochemical factors in mental illness may aid in the development of more effective treatment and prevention of mental illness. Brain derived neurotrophic factor (BDNF) is a widely distributed substance in the central nervous system. It plays an important role in the adult brain, regulating neuronal integrity, promoting synaptic plasticity. It also modulates synthesis, 
metabolism and release of neurotransmitters. BDNF crosses the blood brain barrier (BBB) and serum concentrations strongly correlate with brain levels [1].

Animal models demonstrate stress-induced dysregulation of BDNF-expression, especially in response to chronic stress [2]. In humans, lower serum BDNF levels have been seen in patients with major depressive disorder (MDD), bipolar disorder (BD), schizophrenia, eating disorder, obsessive-compulsive disorder (OCD) and alcohol dependency [2]. In most studies involving MDD, serum BDNF levels correlated negatively with disease severity, and antidepressant treatment increased serum levels of BDNF, as do antipsychotics in schizophrenia [3]. These findings could indicate that a low BDNF level reflects general mental stress. Some studies found that serum BDNF levels were not influenced by neither type of psychotropic medication, nor doses. They found no significant association between antidepressants and BDNF levels, but recommended follow-up studies in drug-naïve patient [2]. Most studies in this field are, however, on distinct diagnostic groups or personality traits compared to healthy controls [2]. These diagnostic groups may include a heterogeneous spectre of symptoms and a specific role of BDNF in specific symptoms has not been described.

C-reactive Protein (CRP) is a well-known biological marker of systemic inflammation. High sensitivity (hs)-CRP is a more sensitive test for subtle inflammation, and serum hs-CRP may reflect a low-grade inflammatory state of various mental disorders [4]. CRP is synthesized in the liver in response to certain pro-inflammatory cytokines [5].

As the psychiatric diagnostic groups are merely syndromal, with collections of symptoms or signs often seen together, they may not represent one common biological process. The diagnostic groups for mental illnesses are wide and partly unspecific, masking differences within the groups [6]. Also, the observed rate of co-occurrence of symptoms among some general psychiatric disorders is high [7], and could in part be due to their multi-factorial nature [8]. Mental suffering and symptoms also may be grouped into "clusters of symptoms" independent of diagnostic groups. One of the most widely replicated disorders are the mood and anxiety disorders [9].

Studying symptoms in relation to biological markers as possible indicators of disease or symptoms may be crucial to reveal causative relations. There are a few reports on altered levels of biological markers in relation to specific symptoms rather than diagnostic groups, e.g. hs-CRP and MCP in fatigue [10] and TGF and aggression / agitation [11], but this field is not extensively studied. To the best of our knowledge, no studies have so far investigated the relation between psychiatric symptoms in patients with known mental disorders and serum BDNF as well as hs-CRP.

The aim of the present study was to explore the associations between psychiatric symptoms as measured by of SCL-90-R with serum BDNF and hs-CRP levels in a patient sample admitted to open ward inpatient stay for treatment of psychiatric disorders. Also, we wanted to compare the group regularly using psychotropic medication and those not using psychotropic medication. 


\section{Methods}

\section{Participants}

In this cross-sectional study a total of 138 patients were recruited from an open in-patient general psychiatric ward, at the Department of Mental health and Addiction, Nordland Hospital Trust, Bodø, Norway. Six participants were excluded due to incomplete data sets.

Patients aged 18 years and above were recruited consecutively in the period February 2014 to February 2018. Patients were referred from the hospital's outpatient services and from general practitioners. Patients not giving their consent and not understanding Norwegian language or otherwise unable to give informed consent were excluded. A research nurse informed eligible patients about the study and written informed consent was obtained by a doctor administering the clinical assessments.

\section{Ethics:}

The study was approved by the Regional Ethics Committee (notification 2015/1809/REK Nord).

\section{Data collection and assessment of psychiatric symptoms}

The following data were obtained from patients: Weight, height and smoking habit.

Age and gender were given from person identification data. All the patients were assessed by the main investigator (first author) upon consultation approximately one week after admittance to the ward. At assessment a rating scale for psychiatric symptoms was administered. Symptom check list 90 (SCL-90$\mathrm{R}$ ) is a validated 90 item rating scale used in research for monitoring actual symptoms and symptom clusters experienced by the patient during the last week. SCL-90 gives a valid picture of mental suffering, and is also considered a useful tool for measuring psychological status, measuring change in outcome studies, or screening for mental disorders. Each of the 90 items is rated on a five-point scale, ranging from, not at all (0) to extremely (4) [12]. The 90 single items are often grouped as primary dimensions or clusters: Depression, somatization, obsessive-compulsive, interpersonal sensitivity, anxiety, angerhostility, phobic-anxiety, paranoid ideation, and psychoticism. All symptom clusters are measured with raw scores in our investigation. Global Severity Index (GSI) is an index that provide measures of overall psychological distress. We stratified the sample in two subgroups: Those using psychotropic medication regularly and those not using psychotropic medication.

\section{Biological measures}

Blood was withdrawn by trained technicians in the morning the day of assessment between 08:00-10:00 a.m., after approximately 12 hours of fasting and rest and no exercise. Biochemical measures were performed at the Department of Laboratory Medicine, Nordland Hospital Trust. For measurement of serum BDNF, blood was withdrawn in Vacuette gel-tubes, left for 30 minutes on ice before centrifugation for 10 minutes at $2300 \times \mathrm{g}(3500 \mathrm{rpm})$. Serum $(2 \times 1 \mathrm{~mL})$ was stored in Matrix tubes on ice up to 2 hours 
before freezing at $-80^{\circ} \mathrm{C}$. Human Free BDNF Quantikine ELISA Kit (R\&D Systems, Minneapolis, MN, USA) was used according to the instructions from the manufacturer and performed by trained technicians. hsCRP in serum was analyzed on a Siemens Prospec nephelometer using CardioPhase ${ }^{\circledR}$ hs-CRP reagents kits from Siemens Healthineers (Erlangen, Germany).

\section{Statistical analysis}

Multiple linear regression analysis was performed using serum BDNF and hs-CRP as dependent variables and symptom clusters as well as confounding factors as BMl, smoking, age and sex as independent variables. The sample were split into two subgroups; patient using psychotropic medication regularly and patients not using psychotropic medication. For all analyses the IBM-SPSS version 26.0 was used, and the statistical significance was set at $p<0.05$.

\section{Results}

\section{Demographics}

The characteristics of participants are presented in Table 1. Among the 132 patients there were 84 women and 48 men with a mean age of 37 years. Patients were recruited over a 4 -year period. A total of 102 patients of the 132 were using psychotropic medication.

\section{Biological measures}

BDNF levels spanned from 13093 to $50434 \mathrm{pg} / \mathrm{mL}$ with a mean of $27184 \mathrm{pg} / \mathrm{mL}$. hs-CRP levels spanned from 0.0 to $77.1 \mathrm{mg} / \mathrm{L}$ with a mean of $3.5 \mathrm{mg} / \mathrm{L}$. When two outliers of hs-CRP $>35 \mathrm{mg} / \mathrm{L}$ were removed the mean was reduced to $2.6 \mathrm{mg} / \mathrm{L}$, Table 1 . 
Table 1

Characteristics of study participants

\begin{tabular}{|lllll|}
\hline & $\mathbf{n}$ & $\%$ & \\
\hline Gender (male) & 47 & 36 & & \\
\hline Smoking & 60 & 46 & & \\
\hline Psychotropic medication & 102 & 77 & & \\
\hline Antidepressant & 87 & 66 & & \\
\hline Antipsychotic & 5 & 4 & 28 & 7 \\
\hline Other & 10 & 8 & 37 & 14 \\
\hline BMI & Min & Max & 6253 \\
\hline Age (years) & 15 & 55 & 27184 & 3 \\
\hline BDNF (pg/ml) & 18 & 78 & 2.7 & \\
hs-CRP & 13093 & 50435 & 14 & \\
\hline BDNF: Normal range: $6186-42580$ pg/mL. hs-CRP: Normal range < 4 mg/L. N= 132. & \\
\hline
\end{tabular}

\section{Psychometrics}

Our participants had highest scores on depression, obsessive- compulsive, somatization, and anxiety symptom clusters in SCL-90, Table 2. 
Table 2

Range of symptom clusters, raw-scores from SCL-90-R

\begin{tabular}{|lllll|}
\hline & N* & Mean & Minimum & Maximum \\
\hline Depression cluster & 125 & 27 & 0 & 46 \\
\hline Somatization cluster & 125 & 19 & 0 & 46 \\
\hline Anxiety cluster & 126 & 17 & 0 & 37 \\
\hline Phobic anxiety cluster & 120 & 10 & 0 & 25 \\
\hline Paranoid ideation cluster & 122 & 7 & 0 & 20 \\
\hline Anger hostility cluster & 128 & 4 & 0 & 21 \\
\hline Psychotisism cluster & 127 & 9 & 0 & 30 \\
\hline Obsessive Compulsive & 126 & 20 & 0 & 35 \\
\hline Interpersonal sensitivity & 126 & 16 & 0 & 22 \\
\hline Global severity index & 127 & 12 & 0 & \\
\hline $\begin{array}{l}\text { *Valid N 95, cases excluded listwise. Cases included in the analysis only if they have full data on all } \\
\text { of the variables listed. }\end{array}$ & & & \\
\hline
\end{tabular}

\section{Multiple linear regression analysis}

BDNF as well as hs-CRP were set as dependent variables and symptom clusters and confounding factors as predictors. We used split file for psychotropic medication and analysed in two subgroups; with or without psychotropic medication. In the regression analysis no co-linearity problem was detected, also evaluated with residual analysis.

In the subgroup of patients not using psychotropic medication, significantly higher BDNF was seen for all SCL-90 symptom clusters apart from anger-hostility, obsessive-compulsive and paranoid ideation. Only the finding for anxiety remained significant also after adjusting for multiple testing. The results remained significant when adjusting for age, gender, smoking and BMI, Table 3 . 
Table 3

Multiple linear regression analysis of BDNF and symptom clusters from group with no psychotropic medication.

\begin{tabular}{|llll|}
\hline & Coefficient & $P$-value* & $95 \%$ Cl \\
\hline Depression & 0.4 & 0.032 & $(115.8-490.3)$ \\
\hline Somatization & 0.4 & 0.028 & $(49.4-504.1)$ \\
\hline Anxiety & 0.6 & 0.004 & $(157.7-641.8)$ \\
\hline Phobic Anxiety & 0.4 & 0.035 & $(151.7-770.9)$ \\
\hline Paranoid Ideation & 0.3 & 0.175 & $(82.8-872.2)$ \\
\hline Anger Hostility & 0.4 & 0.066 & $(110.8-1410.8)$ \\
\hline Psychotisism & 0.5 & 0.031 & $(163.2-764.7)$ \\
\hline Obsessive Compulsive & 0.3 & 0.073 & $(126.9-658.3)$ \\
\hline Interpersonal Sensitivity & 0.4 & 0.025 & $(161.1-710.8)$ \\
\hline Global Severity Index & 0.5 & 0.008 & $(298.9-1123.9)$ \\
\hline $\begin{array}{l}\text { *Adjusted for age, gender, smoking and BMl. BDNF dependent variable, symptom clusters } \\
\text { independent variables. }\end{array}$ & & \\
\hline
\end{tabular}

In the subgroup of patients using psychotropic medication the paranoid ideation cluster was the only symptom cluster positively associated with BDNF, adjusted for age, gender, smoking and BMI, table 4 . 
Table 4

Multiple linear regression analysis of BDNF and symptom clusters from group with psychotropic medication.

\begin{tabular}{|llll|}
\hline & Coefficient & P-value* & $95 \% \mathrm{Cl}$ \\
\hline Depression & -0.03 & 0.750 & $(-136.1-100.4)$ \\
\hline Somatization & -0.10 & 0.357 & $(-163.2-63.6)$ \\
\hline Anxiety & -0.17 & 0.091 & $(-298.8--0.9)$ \\
\hline Phobic -anxiety & -0.15 & 0.161 & $(-320.2-42,7)$ \\
\hline Paranoid Ideation & -0.25 & 0.022 & $(-579.7--39.2)$ \\
\hline Anger- hostility & -0.15 & 0.109 & $(-683.3-22.2)$ \\
\hline Psychotisism & -0.17 & 0.088 & $(-418.9-9.8)$ \\
\hline Obsessive-compulsive & -0.17 & 0.094 & $(-281-16.8)$ \\
\hline Interpersonal sensitivity & -0.16 & 0.124 & $(-298.9-4.8)$ \\
\hline Global severity Index & -0.18 & 0.081 & $(-467.0-77.6)$ \\
\hline $\begin{array}{l}\text { *Adjusted for age, gender, smoking and BMI. Hs-CRP dependent variable, symptom clusters } \\
\text { independent variables }\end{array}$ & & & \\
\hline
\end{tabular}

For hs-CRP no associations with symptom clusters were seen in either group, adjusted for age, gender, smoking and BMI, tables 5 and 6 . 
Table 5

Multiple linear regression analysis of hs-CRP and symptom clusters from group with no psychotropic medication.

\begin{tabular}{|lllc|}
\hline & Coefficient & P-value* & 95\% Cl \\
\hline Depression & 0.1 & 0.724 & $-0.05-3.9$ \\
\hline Somatization & 0.1 & 0.726 & $-0.1-0.1$ \\
\hline Anxiety & -0.1 & 0.639 & $-0.1-0.1$ \\
\hline Phobic -anxiety & 0.4 & 0.058 & $-0.2-0.2$ \\
\hline Paranoid Ideation & 0.3 & 0.275 & $-0.1-0.3$ \\
\hline Anger- hostility & 0.2 & 0.314 & $-0.2-0.4$ \\
\hline Psychotisism & 0.2 & 0.390 & $-0.1-0.2$ \\
\hline Obsessive-compulsive & -0.2 & 0.920 & $-.1-0.2$ \\
\hline Interpersonal sensitivity & 0.3 & 0.192 & $-0.1-0.2$ \\
\hline Global severity Index & 0.2 & 0.272 & $-0.2-0.2$ \\
\hline $\begin{array}{l}\text { *Adjusted for age, gender, smoking and BMI. Hs-CRP dependent variable, symptom clusters } \\
\text { independent variables. }\end{array}$ & & \\
\hline
\end{tabular}

\section{Table 6}

Multiple linear regression analysis of hs-CRP and symptom clusters from group with psychotropic medication.

\begin{tabular}{|lllc|}
\hline & Coefficient & P-value* & $95 \% \mathrm{Cl}$ \\
\hline Depression & -0.03 & 0.752 & $-0.5-0.1$ \\
\hline Somatization & 0.02 & 0.863 & $-0.3-0.1$ \\
\hline Anxiety & 0.09 & 0.396 & $-0.0-0.1$ \\
\hline Phobic -anxiety & -0.12 & 0.288 & $-0.1-0.1$ \\
\hline Paranoid Ideation & -0.18 & 0.101 & $-0.1-0.1$ \\
\hline Anger- hostility & -0.06 & 0.595 & $-0.1-0.2$ \\
\hline Psychotisism & -0.08 & 0.445 & $0.1-0.2$ \\
\hline Obsessive-compulsive & -0.17 & 0.100 & $-0.1-0.1$ \\
\hline Interpersonal sensitivity & -0.13 & 0.211 & $-0.1-0.1$ \\
\hline Global severity Index & -0.04 & 0.720 & $-0.1-0.2$ \\
\hline $\begin{array}{l}* \text { Adjusted for age, gender, smoking and BMI. Hs-CRP dependent variable, symptom clusters } \\
\text { independent variables. }\end{array}$ & & \\
\hline
\end{tabular}




\section{Discussion}

After correcting for confounders, significant positive associations between BDNF and most symptom clusters; depression, somatization, interpersonal sensitivity, anxiety, phobic anxiety, and psychoticism, as well as a Global severity Index were found in the subgroup of patient not using psychotropic medication. For the patient group taking psychotropic medication a positive association between BDNF and symptom clusters was seen only for the paranoid ideation cluster. Regarding hs-CRP we did not find any association with symptom clusters in any of the groups.

BDNF is suggested as a biomarker in mental disorders [13]. For the unmedicated patient group we found a positive association between level of BDNF and intensity of symptoms in several SCL-90 clusters. In line with our findings a recent study reported higher levels of BDNF in bipolar disorder, where higher levels of BDNF were associated with longer illness duration [14]. Some studies found significant relation of BDNF with negative symptoms in schizophrenia and it has been suggested that the increased level of BDNF represents a compensatory response to the underlying neuropathology in the brain regions implicated [15]. Our findings also are in keeping with the findings of Kheirouri et al [16] in patients with major depressive disorder (MDD).

However, most previous studies report a negative association between mental suffering and level of BDNF. This negative association is reported to reverse with successful treatment [2]. Our findings of significant positive associations between BDNF and most symptom clusters in non-medicated patients including depression, somatization, anxiety and psychoticism may be explained through the involvement of BDNF in the hypothalamic-pituitary-adrenal (HPA) axis in depression: The psychological stress of depression, causes an upregulated level of BDNF that in turn causes an increase in corticotrophinreleasing hormone leading to glucocorticoid release [16]. The findings need to be repeated.

Reasons why we did not see the pattern with inverse association between psychiatric symptoms and BDNF that has been reported in many other studies, may be related to our patient population and the assessment and sampling one week after admittance. At the time of assessment patients are expected to be improving from their mental suffering. One explanation may be that the body and brain conduct selfrepair and compensates by upregulating BDNF. The group of patients using psychotropic medication did not show this upregulation despite the same symptom burden as reported by SCL-90-R.

We did not find any significant association between symptom clusters and hs-CRP when adjusted for confounding factors. Other studies have found that hs-CRP is related to severity of depressive symptoms [17]. Increased hs-CRP has been reported in patients with fibromyalgia and chronic fatigue syndrome [10] as well as in depression and anxiety [18]. We do not have a group of healthy controls and thus cannot claim that our patients actually have increased hs-CRP, but a mean level 2.7 still represent moderately increased risk of cardiovascular disease (low risk cut off $<1 \mathrm{mg} / \mathrm{L}$ ).

Overall, we could not reproduce the findings from other studies in hs-CRP, and the findings on BDNF may seem in conflict with previous reports. Factors contributing to inconsistency in this field may be variations 
in methodology, symptom severity, medication, measure used to diagnose and size and ethnicity of the sample [19]. Previous studies have demonstrated associations between serum BDNF and lifestyle factors as smoking, BMI and physical activity [20], as well use of psychotropic medication [2]. It is also known that age and gender can affect the BDNF level. A higher BDNF serum level is associated with increasing age, residence in high degree of urbancy and smoking [19].

There are several limitations to the study. Information on symptoms, smoking, weight and height in our study were obtained from the self-report questionnaire with a risk of biased reporting. The sample size is small, especially regarding the subgroup with no use of psychotropic medication. However, other similar studies published in this field report smaller numbers $(n<100)$ [13], [16], [19]. Our aim was to search for association between BDNF and mental suffering in the two groups; with or without psychotropic medication. We did not study BDNF in relation to diagnoses or symptoms compared to a healthy control group, and this is a main limitation. However, we are still able to compare general mental suffering with BDNF and hsCRP adjusting for the known confounding factors. We do not use psychiatric diagnostic groups in this study, thus we cannot relate the findings directly to other studies based on diagnostic groups. Also, this is a cross sectional natural study. Patients were not randomised to either medication or non-medication. Furthermore, as mentioned they were not acutely ill. One main originality of our study is the focus on symptoms and symptom clusters rather than diagnostic groups. Another original factor in our study is that the data were sampled roughly 7 days after admittance to reduce potential stress achieved by the admittance itself for the patients and thereby to reduce any possible stress-induced alterations in the biological markers. During these 7 days patients will also have been exposed to care, safety, professional milieu therapy, psychotherapy, often improved sleep and nutrition. All of which could contribute to reduce the possible stress of admittance to the inpatient setting. Also, the focus on a general open ward inpatient group may add information to the field regarding effect of different degrees of illness compared to patient groups in other studies.

There also are several general strengths. We adjusted for smoking, weight and height as well as use of psychotropic medication. Regarding biological measures, serum was sampled after rest and fasting in the morning to avoid effects of diurnal variation. As serum BDNF levels have been shown to be significantly lower if blood is drawn in the afternoon and diurnal variations of BDNF plasma levels are known [20] we sampled sera fasting in the morning, and without previous exercise. Furthermore, the laboratory used a standardized protocol for analysis and with an experienced technician performing the analysis.

\section{Conclusion}

Our findings revealed that contrary to most previous reports, serum BDNF levels were positively associated with several psychiatric symptoms in the group of patients not using psychotropic medication. This was not seen in the group on psychotropic medication. We speculate that the group of patients not using psychotropic medication depict more biological alteration than those on medication, and that upregulation of BDNF has occurred in the medication free group. Patients using psychotropic 
medication are probably not in need of the same upregulation because the medication are modulating the underlying biological pathology, but this needs further investigation.

hs-CRP had a mean level of $2.7 \mathrm{mg} / \mathrm{L}$ which may represent a moderately increased risk of cardiovascular disease [21], but no associations were found to the symptom clusters. This is in line with the findings in other studies that increased hs-CRP is not specific for a certain symptom cluster, but rather related to mental suffering and cardiovascular risk in general.

The major contribution of the present study is the use of symptoms rather than diagnosis, the integration of confounding factors, and standardized analysis of serum BDNF. To our knowledge this is the only report on the relationship between psychiatric symptoms, BDNF and hs-CRP in two groups; with and without psychotropic medication use in a sample with inpatients with general mental disorders from Northern Norway.

Our results provide further evidence for a role for BDNF in the pathophysiology of mental disorders, especially revealed in the group of patients not using psychotropic medication. Furthermore, it also suggests that BDNF alteration in general mental disorder reflect response to general stress and mental illness, independent of presented symptoms.

\section{Abbreviations}

BDNF, Brain Derived neurotropic Factor; BMI, Body Mass Index; Cl, confidence interval; hs-CRP, high sensitive C-Reactive protein; SCL-90-R, Symptom Check List Revised; SPSS, Statistical Package for the Social Sciences

\section{Declarations}

\section{Funding}

This study was funded in part by a grant from Northern Norway Regional Health Authority.

\section{Availability of data/materials}

The datasets used and analysed during the current study available from the corresponding author on reasonable request.

\section{Author contributions}

HSN wrote the outline and the first draft of the article. All authors contributed to, and have approved the final manuscript.

\section{Competing interests}

The authors declared no conflicts of interests with respect to their authorship or publication of this article. 
Ethical approval and concent to participate

The study was approved by the Regional Ethics Committee, notification 2015/1809/REK Nord. All methods were performed in accordance with the relevant guidelines and regulations.

\section{Aknowledgments}

We would like to thank Lill Magna Lekanger for preparing the data files as well as Hilde Myrvold and AnnTorill Kokaas for patient coordination upon admittance. Judith Krey Ludviksen is also greatly acknowledged for analyzing BDNF and Evy Eide for analyzing hs-CRP. We also thank Erling Kvig and Francis Odeh for counselling during the process, Nina Groven for the advice and counselling with SPSS and data files, as well as Martin Soloey Myrland for assistance in making tables.

\section{References}

1. Toll A, Mane A: Brain-derived neurotrophic factor levels in first episode of psychosis: A systematic review. World J Psychiatry 2015, 5(1):154-159.

2. Nomoto H, Baba H, Satomura E, Maeshima H, Takebayashi N, Namekawa Y, Suzuki T, Arai H: Serum brain-derived neurotrophic factor levels and personality traits in patients with major depression. $B M C$ Psychiatry 2015, 15:33.

3. Fernandes BS, Berk M, Turck CW, Steiner J, Goncalves CA: Decreased peripheral brain-derived neurotrophic factor levels are a biomarker of disease activity in major psychiatric disorders: a comparative meta-analysis. Mol Psychiatry 2014, 19(7):750-751.

4. Kim JR, Kim HN, Song SW: Associations among inflammation, mental health, and quality of life in adults with metabolic syndrome. Diabetol Metab Syndr 2018, 10:66.

5. Sproston NR, Ashworth JJ: Role of C-Reactive Protein at Sites of Inflammation and Infection. Front Immunol 2018, 9:754.

6. Bebbington P: Categories, continua and the growth of psychiatric knowledge. Soc Psychiatry Psychiatr Epidemio/2015, 50(4):507-510.

7. Hyman SE: Grouping diagnoses of mental disorders by their common risk factors. Am J Psychiatry 2011, 168(1):1-3.

8. Lakhan SE, Vieira K, Hamlat E: Biomarkers in psychiatry: drawbacks and potential for misuse. Int Arch Med 2010, 3:1.

9. Oiesvold T, Nivison M, Hansen V, Skre I, Ostensen L, Sorgaard KW: Diagnosing comorbidity in psychiatric hospital: challenging the validity of administrative registers. BMC Psychiatry 2013, 13:13.

10. Groven N, Fors EA, Reitan SK: Patients with Fibromyalgia and Chronic Fatigue Syndrome show increased hsCRP compared to healthy controls. Brain Behav Immun 2019, 81:172-177.

11. Larsen JB, Stunes AK, Vaaler A, Reitan SK: Cytokines in agitated and non-agitated patients admitted to an acute psychiatric department: A cross-sectional study. PLoS One 2019, 14(9):e0222242. 
12. Schmitz N, Hartkamp N, Kiuse J, Franke GH, Reister G, Tress W: The Symptom Check-List-90-R (SCL90-R): a German validation study. Qual Life Res 2000, 9(2):185-193.

13. Skibinska M, Kapelski P, Rajewska-Rager A, Pawlak J, Szczepankiewicz A, Narozna B, TwarowskaHauser J, Dmitrzak-Weglarz M: Brain-derived neurotrophic factor (BDNF) serum level in women with first-episode depression, correlation with clinical and metabolic parameters. Nord J Psychiatry 2018, 72(3):191-196.

14. Munkholm K, Vinberg M, Kessing LV: Peripheral blood brain-derived neurotrophic factor in bipolar disorder: a comprehensive systematic review and meta-analysis. Mol Psychiatry 2016, 21(2):216228.

15. Binford SS, Hubbard EM, Flowers E, Miller BL, Leutwyler H: Serum BDNF Is Positively Associated With Negative Symptoms in Older Adults With Schizophrenia. Biol Res Nurs 2018, 20(1):63-69.

16. Kheirouri S, Noorazar SG, Alizadeh M, Dana-Alamdari L: Elevated Brain-Derived Neurotrophic Factor Correlates Negatively with Severity and Duration of Major Depressive Episodes. Cogn Behav Neurol 2016, 29(1):24-31.

17. Kohler-Forsberg O, Buttenschon HN, Tansey KE, Maier W, Hauser J, Dernovsek MZ, Henigsberg N, Souery D, Farmer A, Rietschel M et al: Association between C-reactive protein (CRP) with depression symptom severity and specific depressive symptoms in major depression. Brain Behav Immun 2017, 62:344-350.

18. Tayefi M, Shafiee M, Kazemi-Bajestani SMR, Esmaeili H, Darroudi S, Khakpouri S, Mohammadi M, Ghaneifar Z, Azarpajouh MR, Moohebati M et al: Depression and anxiety both associate with serum level of hs-CRP: A gender-stratified analysis in a population-based study. Psychoneuroendocrinology 2017, 81:63-69.

19. Elfving B, Buttenschon HN, Foldager L, Poulsen PH, Andersen JH, Grynderup MB, Hansen AM, Kolstad HA, Kaerlev L, Mikkelsen S et al: Depression, the Val66Met polymorphism, age, and gender influence the serum BDNF level. J Psychiatr Res 2012, 46(9):1118-1125.

20. Bus BA, Molendijk ML, Penninx BJ, Buitelaar JK, Kenis G, Prickaerts J, Elzinga BM, Voshaar RC: Determinants of serum brain-derived neurotrophic factor. Psychoneuroendocrinology 2011, 36(2):228-239.

21. Ridker PM, Rifai N, Rose L, Buring JE, Cook NR: Comparison of C-reactive protein and low-density lipoprotein cholesterol levels in the prediction of first cardiovascular events. N Engl J Med 2002, 347(20):1557-1565. 